\title{
SESQUITERPENOS DE SEMENTES DE Guarea guidonia (MELIACEAE)
}

\section{Luzinátia Ramos Soares}

Departamento de Química, Universidade Federal de Mato Grosso do Sul, CP 549, 79070-900 Campo Grande - MS / Universidade Estadual de Mato Grosso do Sul, Unidade Universitária de Coxim, 79400-000 Coxim - MS, Brasil

Alene Cortes de Queiroz e Silva, Talita Vilalva Freire, Fernanda Rodrigues Garcez e Walmir Silva Garcez*

Departamento de Química, Universidade Federal de Mato Grosso do Sul, CP 549, 79070-900 Campo Grande - MS, Brasil

Recebido em 28/3/11; aceito em 1/7/11; publicado na web em 19/8/11

SESQUITERPENES FROM THE SEEDS OF Guarea guidonia (MELIACEAE). Two new sesquiterpenes $6 \alpha$-ethoxyeudesm-

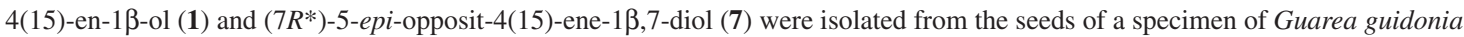
(Meliaceae). Five other known sesquiterpenes, eudesm-4(15)-ene-1 $\beta, 6 \alpha$-diol (2), 5-epi-eudesm-4(15)-ene-1 $\beta, 6 \beta$-diol (3), eudesm4(15)-ene-1 $\beta, 5 \alpha$-diol (4), eudesm-4(15),7-dien-1 $\beta$-ol (5) and (7R*)-opposit-4(15)-ene-1 $\beta, 7$-diol (6), were also isolated in this work. Their structures were determined on the basis of spectral data (MS, IR, and NMR-1D and 2D) and subsequent comparisons with the literature. The substances 3, $\mathbf{4}$ and $\mathbf{6}$ are reported for the first time in Meliaceae and $\mathbf{5}$ for the first time in the genus Guarea.

Keywords: Guarea guidonia; Meliaceae; eudesmane.

\section{INTRODUÇÃO}

Guarea guidonia L. Sleumer (sin. Guarea trichilioides L.), pertencente à família Meliaceae e popularmente conhecida como carrapeta-verdadeira, é uma árvore ocorrente no Brasil desde a região Amazônica até o estado do Rio Grande do Sul, ${ }^{1}$ podendo atingir $20 \mathrm{~m}$ de altura. Na medicina popular é utilizada no tratamento de reumatismo, hidropsia e tumores artríticos, tendo, ainda, propriedades adstringentes, purgativas, febrífugas, abortivas, eméticas e anti-inflamatórias. ${ }^{1}$

Estudos fitoquímicos prévios dos extratos hexânico e etanólico da casca do tronco desta espécie, além do óleo volátil de suas folhas, resultaram no isolamento de limonoides, ${ }^{2-3}$ esteroides, ${ }^{4}$ seco-esteroides,${ }^{5}$ cumarinas, ${ }^{2}$ di- ${ }^{4,6,7}$ tri- ${ }^{-4}$ e sesquiterpenos..$^{2,4,8}$

O presente trabalho trata do estudo fitoquímico de sementes de um espécime de G. guidonia, que levou ao isolamento de sete sesquiterpenos, sendo dois deles inéditos: $6 \alpha$-etoxieudesm-4(15)-eno-1 $\beta$-ol (1), eudesm-4(15)-eno-1 $\beta, 6 \alpha$-diol (2), 5-epi-eudesm-4(15)-eno$1 \beta, 6 \beta$-diol (3), eudesm-4(15)-eno-1 $\beta, 5 \alpha$-diol (4), eudesm-4(15),7dien-1 $\beta$-ol (5), (7R*)-oposit-4(15)-eno-1 $\beta, 7$-diol (6) e (7R*)-5-epioposit-4(15)-eno-1 $\beta, 7$-diol (7) (Figura 1). As substâncias 1 e 7 são inéditas na literatura, enquanto 3, 4 e $\mathbf{6}$ estão sendo descritas pela primeira vez em Meliaceae e $\mathbf{5}$ pela primeira vez no gênero Guarea.

\section{PARTE EXPERIMENTAL}

\section{Instrumentação e procedimentos gerais}

Para cromatografia em camada fina utilizaram-se placas de gel de sílica (Whatman), com indicador de fluorescência $\mathrm{UV}_{254}$, em camadas de 0,25 $\mathrm{mm}$ de espessura, empregando-se como revelador solução saturada de sulfato de cério IV em ácido sulfúrico $36 \%$. A separação cromatográfica em coluna foi realizada utilizando-se gel de sílica 230-400 mesh (Acros Organics). Os experimentos de RMN ${ }^{1} \mathrm{H}$ $\mathrm{e}^{13} \mathrm{C}$ (uni- e bidimensionais) foram obtidos em espectrômetro Bruker DPX-300 (300/75 MHz), utilizando-se $\mathrm{CDCl}_{3}$ como solvente e o sinal do $\mathrm{CHCl}_{3}$ residual como referência. Os espectros de IV foram

\footnotetext{
*e-mail: walmir.garcez@ufms.br
}

registrados em espectrômetro Bomem-Hartmann \& Braun FT, tendo sido as amostras preparadas sob a forma de filme. As medidas de rotação óptica foram determinadas em polarímetro Perkin-Elmer 341 e os espectros de massas obtidos no espectrômetro GCMS-QP2010 Plus, Shimadzu.

\section{Material vegetal}

O material vegetal foi coletado no distrito de Palmeiras, município de Dois Irmãos do Buriti - MS. A identificação botânica foi realizada pela MSc. U. M. Resende (DBI/UFMS); uma exsicata (número 11217) foi depositada no Herbário CGMS, da UFMS.

\section{Extração e isolamento das substâncias}

As sementes frescas $(398,2 \mathrm{~g})$ foram trituradas e submetidas à extração com etanol, à temperatura ambiente, por 5 dias. A solução resultante após filtração foi concentrada sob pressão reduzida, fornecendo o extrato etanólico bruto $(37,0 \mathrm{~g})$. Este foi submetido a partições líquido-líquido entre metanol/água (9:1) e hexano, seguido por acetato de etila, dando origem a três fases: hexânica $(24,5 \mathrm{~g})$, acetato de etila $(4,5 \mathrm{~g})$ e hidrometanólica $(6,5 \mathrm{~g})$.

O material obtido após a evaporação do acetato de etila (4,5 g) foi submetido a uma extração com clorofórmio, tendo o material solúvel neste originado um resíduo com 3,8 g. Coluna cromatográfica deste resíduo, utilizando gel de sílica 230-400 mesh (122 g), eluída com mistura de Hex-AcOEt nas proporções de 95:5 e 90:10, originou 850 frações de $5 \mathrm{~mL}$ cada. Este processo, que foi monitorado por cromatografia em camada fina de sílica-gel (Hex-AcOEt 80:20 e 70:30), resultou no isolamento das substâncias $\mathbf{1}$ (3,8 mg), 2 (4,2 mg), 3 (7,6 $\mathrm{mg}), \mathbf{4}(3,6 \mathrm{mg}), \mathbf{5}(6,9 \mathrm{mg}), \mathbf{6}(18,6 \mathrm{mg})$ e $\mathbf{7}(4,3 \mathrm{mg})$.

6 $\alpha$-etoxieudesm-4(15)-en-1 $\beta$-ol (1)

Óleo amarelo, $[\alpha]_{\mathrm{D}}{ }^{23}+9,7^{\circ}\left(\mathrm{CHCl}_{3}\right.$; conc. 0,19$)$. $\mathrm{RMN}{ }^{1} \mathrm{H} \mathrm{e}^{13} \mathrm{C}$ : Tabela 1.

Eudesm-4(15)-eno-1 $\beta, 6 \alpha$-diol (2)

Óleo amarelo, $[\alpha]_{\mathrm{D}}{ }^{23}+2,6^{\circ}\left(\mathrm{CHCl}_{3}\right.$; conc. 0,20$)$. $\mathrm{RMN}{ }^{1} \mathrm{H}(300$ $\left.\mathrm{MHz}, \mathrm{CDCl}_{3}\right) \delta_{\mathrm{H}}$ (mult.; $J$ em Hz; H): 3,40 (dd; 11,5 e 4,7; H-1), 

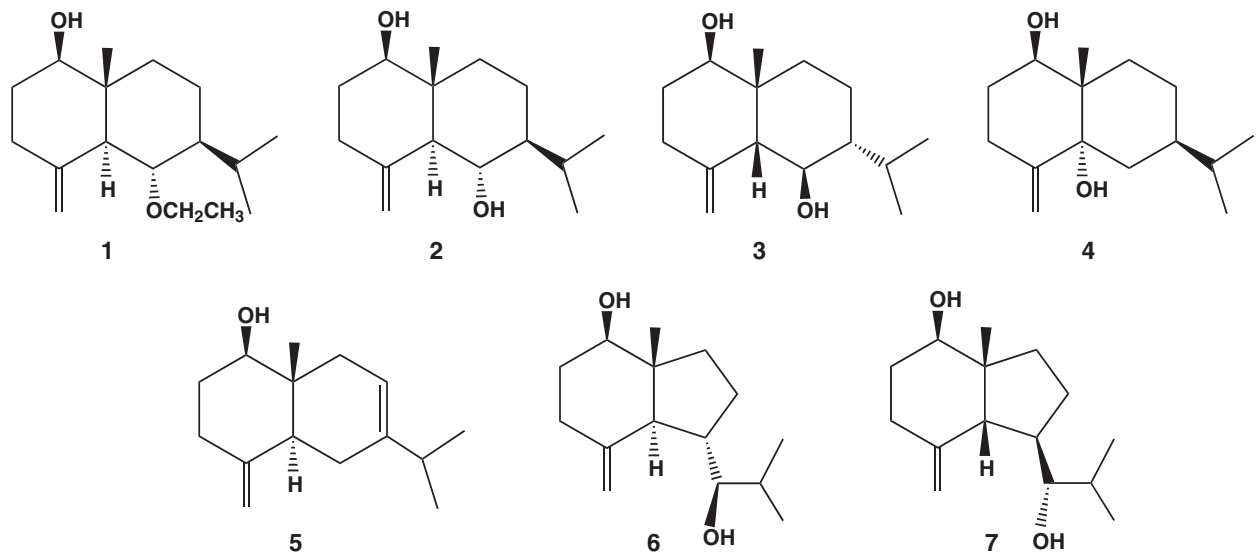

Figura 1. Estruturas das substâncias 1-7

$2,04(t d ; 13,3$ e 5,$1 ; \mathrm{H}-3 \alpha), 2,33$ ( $d d d ; 13,3,5,1$ e 2,3; H-3ß), 3,69 ( $t$; 9,7; H-6), 2,21 (septd; 7,0 e 2,4; H-11), 0,93 (d; 7,0; H-12), 0,84 (d; 7,0; H-13), 0,68 ( $s$ H-14), 4,72 (d; 2,7; H-15a), 5,00 (d; 2,7; H15b). $\mathrm{RMN}{ }^{13} \mathrm{C}\left(75 \mathrm{MHz}, \mathrm{CDCl}_{3}\right) \delta_{\mathrm{C}}: 79,0(\mathrm{C}-1), 31,9(\mathrm{C}-2), 35,1$ (C-3), 146,2 (C-4), 55,9 (C-5), 67,0 (C-6), 49,3 (C-7), 18,1 (C-8), 36,3 (C-9), 41,7 (C-10), 26,0 (C-11), 21,1 (C-12), 16,2 (C-13), 11,6 (C-14), 107,8 (C-15).

\section{5-Epi-eudesm-4(15)-eno-1 $\beta, 6 \beta$-diol (3)}

Óleo amarelo, $[\alpha]_{\mathrm{D}}{ }^{23}-6,7^{\circ}\left(\mathrm{CHCl}_{3}\right.$; conc. 0,38$)$. RMN ${ }^{1} \mathrm{H}(300$ $\left.\mathrm{MHz}, \mathrm{CDCl}_{3}\right) \delta_{\mathrm{H}}$ (mult.; $J$ em Hz; H): 3,96 (dd; 11,5 e 4,9; H-1), 2,28 ( $m$; H-3a), 2,28 ( $m$; H-3b), 3,53 ( $t$; 9,8; H-6), 2,07 ( $t d ; 13,9$ e 3,1 ; H-9a), 1,04 (td; 13,9 e 4,5; H-9b), (septd; 6,6 e 2,5; H-11), 0,96 (d; 6,8; H-12), 0,86 ( $d ; 6,8 ; \mathrm{H}-13), 0,88$ ( $s$ H-14), 4,85 (sl; H-15a), 4,99 (sl; H-15b). RMN ${ }^{13} \mathrm{C}\left(75 \mathrm{MHz}, \mathrm{CDCl}_{3}\right) \delta_{\mathrm{C}}: 68,2(\mathrm{C}-1), 31,1$ (C-2), 29,7 (C-3), 145,4 (C-4), 61,6 (C-5), 67,0 (C-6), 49,1 (C-7), 18,0 (C-8), 34,4 (C-9), 40,1 (C-10), 26,4 (C-11), 20,9 (C-12), 16, 2 (C-13), 21,3 (C-14), 114,2 (C-15).

\section{Eudesm-4(15)-eno-1 $\beta, 5 \alpha$-diol (4)}

Óleo amarelo, $[\alpha]_{\mathrm{D}}{ }^{23}+8,1^{\circ}\left(\mathrm{CHCl}_{3}\right.$; conc. 0,18$)$. $\mathrm{RMN}{ }^{1} \mathrm{H}(300$ $\left.\mathrm{MHz}, \mathrm{CDCl}_{3}\right) \delta_{\mathrm{H}}$ (mult.; $J$ em Hz; H): 4,03 (dd; 11,7 e 5,1; H-1), 2,68 $(t d ; 13,5$ e 5,$7 ; \mathrm{H}-3 \alpha), 2,13$ ( $d d d ; 13,5,4,8$ e 2,$5 ; \mathrm{H}-3 \beta), 0,89$ ( $d ; 7,0$; $\mathrm{H}-12), 0,88$ ( $d$; 7,0; H-13), 0,73 ( $s ; \mathrm{H}-14), 4,73$ ( $s l$; H-15a), 4,85 ( $s l$; $\mathrm{H}-15 b) . \mathrm{RMN}{ }^{13} \mathrm{C}\left(75 \mathrm{MHz}, \mathrm{CDCl}_{3}\right) \delta_{\mathrm{C}}$ : 73,1 (C-1), 30,6 (C-2), 29,8 (C-3), 150,6 (C-4), 76,2 (C-5), 34,3 (C-6), 38,3 (C-7), 23,7 (C-8), 30,0 (C-9), 42,3 (C-10), 32,8 (C-11), 19,7 (C-12), 20,0 (C-13), 12,7 (C-14), 108,7 (C-15).

\section{Eudesm-4(15),7-dien-1 $\beta$-ol (5)}

Óleo amarelo, $[\alpha]_{\mathrm{D}}{ }^{23}+5,5^{\circ}\left(\mathrm{CHCl}_{3}\right.$; conc. 0,35$)$. RMN ${ }^{1} \mathrm{H}(300$ $\mathrm{MHz}, \mathrm{CDCl}_{3}$ ) $\delta_{\mathrm{H}}$ (mult.; $J$ em Hz; H): 3,49 (dd; 11,7 e 4,4; H-1), 2,33 ( $d d d ; 13,3,4,5$ e 2,$9 ; \mathrm{H}-3$ eq $), 1,95$ ( $s l ; \mathrm{H}-6), 5,31$ (dd; 5,4 e 2,1; H-8a), 2,16 (sept; 6,9; H-11), 0,99 (d; 6,8; H-12), 0,99 (d; 6,8; H-13), 0,63 ( $s$; $\mathrm{H}-14), 4,62$ ( $s l ; \mathrm{H}-15 \mathrm{a}), 4,80$ ( $s l ; \mathrm{H}-15 \mathrm{~b}) . \mathrm{RMN}{ }^{13} \mathrm{C}\left(75 \mathrm{MHz}, \mathrm{CDCl}_{3}\right)$ $\delta_{\mathrm{C}}: 79,5$ (C-1), 31,4 (C-2), 34,3(C-3), 148,4 (C-4), 43,0 (C-5), 25,4 (C-6), 141,6 (C-7), 115,7 (C-8), 38,3 (C-9), 38,9 (C-10), 35,0 (C-11), 21,7 (C-12), 21,2 (C-13), 10,3 (C-14), 107,6 (C-15).

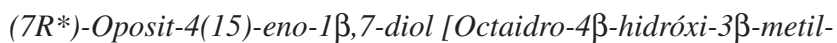
7-metileno- $\alpha$-(1-metiletil)-1H-indeno-1-metanol] (6)

Óleo amarelo, $[\alpha]_{\mathrm{D}}{ }^{23}+2,5^{\circ}\left(\mathrm{CHCl}_{3}\right.$; conc. 0,93$)$. $\mathrm{RMN}{ }^{1} \mathrm{H}(300$ $\left.\mathrm{MHz}, \mathrm{CDCl}_{3}\right) \delta_{\mathrm{H}}$ (mult.; $J$ em Hz; H): 3,56 (dd; 11,3 e 4,9; H-1), 3,21 ( $d d ; 9,7$ e 2,$2 ; \mathrm{H}-7), 0,88$ ( $d ; 6,8 ; \mathrm{H}-12), 0,97$ ( $d ; 6,9 ; \mathrm{H}-13), 0,64$ $(s ; \mathrm{H}-14), 4,79$ ( $d ; 1,3 ; \mathrm{H}-15 \mathrm{a}), 4,92(d ; 1,3 ; \mathrm{H}-15 \mathrm{~b}) . \mathrm{RMN}{ }^{13} \mathrm{C}(75$ $\left.\mathrm{MHz}, \mathrm{CDCl}_{3}\right) \delta_{\mathrm{C}}: 79,0(\mathrm{C}-1), 31,9(\mathrm{C}-2), 34,9(\mathrm{C}-3), 148,9$ (C-4), 56,4
(C-5), 39,4 (C-6), 82,7 (C-7), 26,1 (C-8), 37,3 (C-9), 49,5 (C-10), 31,4 (C-11), 14,7 (C-12), 20,5 (C-13), 12,3 (C-14), 107,6 (C-15).

(7R*)-5-Epi-oposit-4(15)-eno-1 $\beta, 7-d i o l ~[O c t a i d r o-4 \beta-h i d r o ́ x i-3 \alpha$ metil-7-metileno- $\alpha-(1-m e t i l e t i l)-1 H$-indeno-1-metanol] (7)

Óleo amarelo, $[\alpha]_{\mathrm{D}}^{23}-4,7^{\circ}\left(\mathrm{CHCl}_{3}\right.$; conc. 0,17$)$. $\mathrm{RMN}{ }^{1} \mathrm{He} \mathrm{e}^{13} \mathrm{C}$ : Tabela 2.

\section{RESULTADOS E DISCUSSÃO}

A substância 1 apresentou-se como um óleo de coloração amarela e seu espectro IV indicou a presença de grupo hidroxila na estrutura $\left(3420 \mathrm{~cm}^{-1}\right)$. O espectro de RMN ${ }^{1} \mathrm{H}$ de $\mathbf{1}$ (Tabela 1) mostrou sinais relativos a quatro grupos metila $[\delta 0,69(s), 0,84(d, J=7,1 \mathrm{~Hz})$, $0,92(d, J=7,1 \mathrm{~Hz})$ e $1,07(t, J=7,0 \mathrm{~Hz})]$, a hidrogênios olefínicos (dois singletos largos a $\delta 4,83$ e 4,91) e a hidrogênios de um grupo oximetilênico [ $\delta 3,36(d t, J=15,4$ e $7,0 \mathrm{~Hz})$ e $3,32(d t, J=15,4$ e $7,0 \mathrm{~Hz})]$ e dois oximetínicos $[\delta 3,38(d d, J=12,2$ e $5,2 \mathrm{~Hz})$ e 3,55 $(t, J=10,6 \mathrm{~Hz})]$. No espectro de $\mathrm{RMN}{ }^{13} \mathrm{C}$ (Tabela 1$)$ foram listados dezessete sinais, os quais, com o auxílio das informações do espectro DEPT $135^{\circ}$, foram atribuídos a dois carbonos não ligados a hidrogênio (sendo um deles olefínico), cinco metínicos (sendo dois oxigenados), seis metilênicos (sendo um olefínico e um oximetilênico) e quatro metílicos. O espectro de massas de $\mathbf{1}$, que apresentou $\mathbf{M}^{+} \mathrm{em} \mathrm{m} / z, 266$, juntamente com os dados de $\mathrm{RMN}^{13} \mathrm{C}$ indicaram a fórmula molecular $\mathrm{C}_{17} \mathrm{H}_{30} \mathrm{O}_{2}$. Os espectros de $\mathrm{RMN}$ de ${ }^{1} \mathrm{H}$ e ${ }^{13} \mathrm{C}$ indicaram a presença de um grupo $\mathrm{OCH}_{2} \mathrm{CH}_{3}$ e, portanto os quinze carbonos restantes sugeriram que 1 pertence à classe dos sesquiterpenos. A consideração do índice de deficiência de hidrogênios indicou tratar-se de uma estrutura bicíclica e, dentre as possibilidades, os dados do esqueleto carbônico mostraram-se compatíveis com o de um sesquiterpeno tipo eudesmano. Portanto, a proposta inicial para a estrutura da substância 1 foi a de um sesquiterpeno com esqueleto eudesmano, possuindo uma hidroxila secundária, um grupo $\mathrm{O}-\mathrm{CH}_{2} \mathrm{CH}_{3}$ e uma ligação dupla exocíclica $\mathrm{O}$ experimento HSQC evidenciou as correlações que permitiram fazer as atribuições mostradas na Tabela 1. $\mathrm{O}$ experimento HMBC permitiu definir que o grupo etoxila estava ligado ao carbono metínico referente aos sinais $\delta_{\mathrm{C}} 74,4 / \delta_{\mathrm{H}} 3,55$. A localização deste grupo em C-6 foi definida pela correlação à longa distância do hidrogênio correspondente ao sinal a $\delta_{\mathrm{H}} 3,55 \mathrm{com}$ os carbonos metínicos a $\delta_{\mathrm{C}} 51,8$ e 47,0 atribuídos, respectivamente, a C-5 e C-7. A definição da posição da hidroxila secundária foi feita principalmente pela correlação no experimento HMBC entre o hidrogênio a $\delta_{\mathrm{H}} 3,38$ com o carbono quaternário a $\delta_{\mathrm{C}} 42,1$ (C-10) e o metileno a $\delta_{\mathrm{C}} 32,1(\mathrm{C}-2)$. Das duas possibilidades para a ligação dupla, a localização na isopropila foi descartada em função dos dois 
dubletos referentes a duas metilas observados no espectro de RMN ${ }^{1} \mathrm{H}$, sendo, portanto, definida em C-4/C-15. A orientação equatorial ( $\beta$ ) da hidroxila de $\mathrm{C}$-1 foi definida pela constante de acoplamento do sinal de $\mathrm{H}-1\left(\delta_{\mathrm{H}} 3,38, d d, J=12,2\right.$ e $\left.5,2 \mathrm{~Hz}\right)$, sendo confirmada pela correlação observada no experimento NOESY entre $\mathrm{H}-5 / \mathrm{H}-1$. Da mesma maneira, o tripleto de $J=10,6 \mathrm{~Hz}$ referente a H-6 indica dois acoplamentos trans diaxial e, portanto, que o grupo etoxila se encontra equatorialmente orientado. Assim, estrutura de $\mathbf{1}$ foi elucidada como sendo $6 \alpha$-etoxieudesm-4(15)-en-1 $\beta$-ol, que corresponde a uma substância até então não relatada na literatura.

Tabela 1. Dados de $\mathrm{RMN}^{1} \mathrm{H}\left(\mathrm{CDCl}_{3}, 300 \mathrm{MHz}\right)$ e ${ }^{13} \mathrm{C}(75 \mathrm{MHz})$ de $\mathbf{1}$

\begin{tabular}{|c|c|c|c|}
\hline $\mathrm{C} / \mathrm{H}$ & $\delta_{\mathrm{C}}$ & $\delta_{\mathrm{H}}$ & $\mathrm{HMBC}(\mathrm{H} \rightarrow \mathrm{C})$ \\
\hline 1 & 79,5 & $3,38(1 \mathrm{H} ; d d, 12,2$ e $5,2 \mathrm{~Hz})$ & $\begin{array}{l}\text { C-10; C-2; } \\
\text { C-14; C-9 }\end{array}$ \\
\hline \multirow[t]{2}{*}{2} & 32,1 & $1,81(1 \mathrm{H \alpha} ; d t d, 12,2,5,2$ e $2,3 \mathrm{~Hz})$ & - \\
\hline & & $1,51(1 \mathrm{H} \beta ; q d, 12,2$ e $5,2 \mathrm{~Hz})$ & - \\
\hline \multirow[t]{2}{*}{3} & 35,1 & $2,26(1 \mathrm{H} ; d d d, 13,2,5,2$ e $2,3 \mathrm{~Hz})$ & $\mathrm{C}-4 ; \mathrm{C}-15$ \\
\hline & & $2,04(1 \mathrm{H} ; d d d, 13,2,12,2$ e $5,2 \mathrm{~Hz})$ & - \\
\hline 4 & 144,7 & - & - \\
\hline 5 & 51,8 & $1,90(1 \mathrm{H} ; d l, 10,6 \mathrm{~Hz})$ & $\begin{array}{c}\text { C-4; C-15; } \\
\text { C-10 }\end{array}$ \\
\hline 6 & 74,4 & $3,55(1 \mathrm{H} ; t, 10,6 \mathrm{~Hz})$ & $\begin{array}{c}\mathrm{OCH}_{2} \mathrm{CH}_{3} ; \\
\mathrm{C}-5 ; \mathrm{C}-7\end{array}$ \\
\hline 7 & 47,0 & $1,45(1 \mathrm{H} ; t d, 10,6$ e $3,0 \mathrm{~Hz})$ & - \\
\hline \multirow[t]{2}{*}{8} & 18,4 & $1,46(1 \mathrm{H} \beta ; d d t, 13,1,10,6$ e $3,0 \mathrm{~Hz})$ & - \\
\hline & & $1,17(1 \mathrm{H \alpha} ; d q, 13,1$ e $3,0 \mathrm{~Hz})$ & - \\
\hline \multirow[t]{2}{*}{9} & 36,4 & $1,86(1 \mathrm{H} \beta ; d t, 13,1$ e $3,0 \mathrm{~Hz})$ & $\mathrm{C}-10$ \\
\hline & & $1,05(1 \mathrm{H \alpha} ; t d, 13,1$ e $3,0 \mathrm{~Hz})$ & - \\
\hline 10 & 42,1 & - & - \\
\hline 11 & 25,7 & $2,10(1 \mathrm{H} ; \operatorname{sextd}, 7,1$ e $3,0 \mathrm{~Hz})$ & - \\
\hline 12 & 16,0 & $0,84(3 \mathrm{H} ; d, 7,1 \mathrm{~Hz})$ & $\begin{array}{c}\text { C- } 11 ; \mathrm{C}-7 ; \\
\text { C-13 }\end{array}$ \\
\hline 13 & 21,3 & $0,92(3 \mathrm{H} ; d, 7,1 \mathrm{~Hz})$ & $\begin{array}{c}\text { C-11; C-7; } \\
\text { C-12 }\end{array}$ \\
\hline 14 & 11,7 & $0,69(3 \mathrm{H} ; s)$ & $\begin{array}{l}\text { C-1; C-5; } \\
\text { C-10; C-9 }\end{array}$ \\
\hline \multirow[t]{2}{*}{15} & 108,9 & $4,91(1 \mathrm{H} ; s l)$ & $\mathrm{C}-5 ; \mathrm{C}-3$ \\
\hline & & $4,83(1 \mathrm{H} ; s l)$ & $\mathrm{C}-5 ; \mathrm{C}-3$ \\
\hline \multirow[t]{2}{*}{$-\mathrm{OCH}_{2}^{-}$} & 60,1 & $3,36(1 \mathrm{H} ; d t, 15,4$ e $7,0 \mathrm{~Hz})$ & $\underset{\mathrm{C}-6}{\mathrm{OCH}_{2}} \underset{\mathrm{C}}{\mathrm{C}_{3}} ;$ \\
\hline & & $3,32(1 \mathrm{H} ; d t, 15,4$ e $7,0 \mathrm{~Hz})$ & - \\
\hline \multicolumn{2}{|c|}{$\mathrm{OCH}_{2} \mathrm{CH}_{3} \quad 15,8$} & $1,07(3 \mathrm{H} ; t, 7,0 \mathrm{~Hz})$ & $\mathrm{OCH}_{2} \mathrm{CH}_{3}$ \\
\hline
\end{tabular}

As substâncias 2-6 (Figura 1) foram identificadas através das análises dos espectros de $\mathrm{RMN}{ }^{1} \mathrm{H} \mathrm{e}{ }^{13} \mathrm{C}$, seguidas pela comparação com dados descritos na literatura, como sendo os sesquiterpenos eudesm4(15)-eno-1 $\beta, 6 \alpha$-diol (2), 5-epi-eudesm-4(15)-eno-1 $\beta, 6 \beta$-diol (3), eudesm-4(15)-eno-1 $\beta, 5 \alpha$-diol (4), eudesm-4(15),7-dien-1 $\beta$-ol (5) e (7R*)-oposit-4(15)-eno-1 $\beta, 7$-diol (6).

O composto 2 já havia sido caracterizado nas cascas de Guarea guidonia ${ }^{2}$ e a substância 3 foi obtida de uma hepática ${ }^{9}$ e de plantas superiores. ${ }^{10}$ A substância 4, conhecida como Carainterol, também foi isolada de plantas ${ }^{11} \mathrm{e}$, recentemente, foi sintetizada, ${ }^{12}$ enquanto a substância $\mathbf{5}$ tem sido encontrada frequentemente em óleos essenciais. ${ }^{13,14}$ A substância $\mathbf{6}$, que possui o esqueleto pouco comum do tipo opositano, foi obtida anteriormente de plantas dos gêneros Eringeron, ${ }^{11}$ Senecio ${ }^{15}$ e Tephrosia. ${ }^{16}$ No entanto, este é o primeiro registro de isolamento das substâncias 3-6 de Meliaceae.

Tabela 2. Dados de $\mathrm{RMN}{ }^{1} \mathrm{H}\left(\mathrm{CDCl}_{3}, 300 \mathrm{MHz}\right)$ e ${ }^{13} \mathrm{C}(75 \mathrm{MHz})$ de 7

\begin{tabular}{|c|c|c|c|}
\hline $\mathrm{C} / \mathrm{H}$ & $\delta{ }^{13} \mathrm{C}$ & $\delta{ }^{1} \mathrm{H}$ & $\operatorname{HMBC}(\mathrm{H} \rightarrow \mathrm{C})$ \\
\hline 1 & 82,6 & $3,69(1 \mathrm{H} ; d d, 11,8$ e $4,3 \mathrm{~Hz})$ & C-9; C-14 \\
\hline \multirow[t]{2}{*}{2} & 32,1 & $1,85(1 \mathrm{H \alpha} ; d d d d, 14,0,4,3,4,3$ e $4,3 \mathrm{~Hz})$ & - \\
\hline & & $1,45(1 \mathrm{H} \beta ; d d d d, 14,0,12,0,11,8$ e $4,6 \mathrm{~Hz})$ & - \\
\hline \multirow[t]{2}{*}{3} & 30,5 & $2,25(1 \mathrm{H \alpha} ; d d d, 13,0,12,0$ e $4,3 \mathrm{~Hz})$ & - \\
\hline & & $2,35(1 \mathrm{H} \beta ; d d d d, 13,0,4,6,4,3$ e $1,7 \mathrm{~Hz})$ & \\
\hline 4 & 150,4 & - & - \\
\hline 5 & 62,0 & $2,15(1 \mathrm{H} ; d d, 10,0$ e $1,7 \mathrm{~Hz})$ & - \\
\hline 6 & 45,2 & $2,32(1 \mathrm{H} \beta ; d d d d, 11,0,11,0,8,5 \mathrm{e} 4,5 \mathrm{~Hz})$ & - \\
\hline 7 & 71,6 & $3,35(1 \mathrm{H} ; d d, 8,5$ e $2,9 \mathrm{~Hz})$ & $\mathrm{C}-5 ; \mathrm{C}-12$ \\
\hline \multirow[t]{2}{*}{8} & 25,8 & $1,83(1 \mathrm{H \alpha} \alpha d d d d, 13,0,11,6,11,0$ e $5,7 \mathrm{~Hz})$ & \\
\hline & & $1,35(1 \mathrm{H} \beta ; d d d d, 13,0,11,7,11,0$ e $2,0 \mathrm{~Hz})$ & - \\
\hline \multirow[t]{2}{*}{9} & 35,8 & $1,94(1 \mathrm{H \alpha} ; d d d, 13,0,11,7$ e $5,7 \mathrm{~Hz})$ & - \\
\hline & & $1,33(1 \mathrm{H} \beta ; d d d, 13,0,11,6$ e $2,0 \mathrm{~Hz})$ & - \\
\hline 10 & 49,0 & - & - \\
\hline 11 & 31,0 & $1,69(1 \mathrm{H} \beta ; d d d, 7,0,6,8$ e $2,9 \mathrm{~Hz})$ & - \\
\hline 12 & 14,8 & $0,82(3 \mathrm{H} ; d, 6.8 \mathrm{~Hz})$ & $\mathrm{C}-7 ; \mathrm{C}-11 ; \mathrm{C}-13$ \\
\hline 13 & 20,3 & $0,93(3 \mathrm{H} ; d, 7,0 \mathrm{~Hz})$ & $\mathrm{C}-11 ; \mathrm{C}-12$ \\
\hline 14 & 18,0 & $0,90(3 \mathrm{H} ; s)$ & C-10; C-9; C-5 \\
\hline \multirow[t]{2}{*}{15} & 112,1 & $4,91(1 \mathrm{Ha} ; t, 1,7 \mathrm{~Hz})$ & $\mathrm{C}-5 ; \mathrm{C}-3$ \\
\hline & & $4,85(1 \mathrm{Hb} ; t, 1,7 \mathrm{~Hz})$ & $\mathrm{C}-5 ; \mathrm{C}-3$ \\
\hline
\end{tabular}

A substância 7 apresentou aspecto oleoso e coloração amarela e seu espectro de IV mostrou uma banda de absorção a $3440 \mathrm{~cm}^{-1}$, correspondente a um grupo hidroxila. Seu espectro de RMN ${ }^{1} \mathrm{H}$ (Tabela 2) apresentou sinais relativos a três grupos metila a $\delta_{\mathrm{H}} 0,82(d, J=6,8$ $\mathrm{Hz}), 0,90(s)$ e $0,93(d, J=7,0 \mathrm{~Hz})$, a dois hidrogênios metilênicos olefínicos a $\delta_{\mathrm{H}} 4,85$ e $4,91(t, J=1,7 \mathrm{~Hz})$ e a dois hidrogênios oximetínicos a $\delta_{\mathrm{H}} 3,35(d d, J=8,5$ e 2,9 Hz) e 3,69 (dd, $J=11,8$ e 4,3 Hz). No espectro de $\mathrm{RMN}{ }^{13} \mathrm{C}$ de 7 (Tabela 2) foram evidenciados quinze sinais, os quais, com auxílio das informações fornecidas pelo espectro DEPT $135^{\circ}$, foram atribuídos a dois carbonos não ligados a hidrogênio (sendo um deles olefínico), cinco metínicos (sendo dois oxigenados), cinco metilênicos (sendo um olefínico) e três metílicos. Estes dados, juntamente com os do espectro de massas, onde se observou o [M $\left.\mathrm{H}_{2} \mathrm{O}\right]^{+} \mathrm{em} \mathrm{m} / \mathrm{z}, 220$, indicaram a fórmula molecular $\mathrm{C}_{15} \mathrm{H}_{26} \mathrm{O}_{2}$ para 7 . A análise dos dados espectrais de 7 mostrou uma grande semelhança com os do sesquitepeno com o esqueleto rearranjado 6. Os experimentos bidimensionais HSQC e HMBC permitiram definir as atribuições mostradas na Tabela 2. Destacam-se no experimento HMBC as correlações entre o hidrogênio correspondente a $\delta_{\mathrm{H}} 3,35(\mathrm{H}-7)$ e os carbonos a $\delta_{\mathrm{C}} 62,0(\mathrm{C}-5)$ e $14,8(\mathrm{C}-12)$ e também entre o hidrogênio referente ao sinal a $\delta_{\mathrm{H}} 3,69(\mathrm{H}-1)$ e os carbonos em $\delta 35,8$ (C-9) e 18,0 (C-14).

Uma diferença marcante entre os valores de $\mathrm{RMN}{ }^{13} \mathrm{C}$ dos compostos 6 e 7 foi o deslocamento químico de C-14, $\delta_{\mathrm{C}} 11,7$ no primeiro e $\delta_{C} 20,3$ no segundo. Estes dados são compatíveis com uma modificação na estereoquímica da junção dos anéis, que seria, portanto, cis no composto 7. Esta proposta é compatível com a correlação observada no experimento NOESY entre H-5 e H-14 e entre $\mathrm{H}-5 / \mathrm{H}-7$, confirmando as estereoquímicas destes carbonos conforme mostrado na Figura 1. 
A estereoquímica relativa de C-7 foi determinada por comparação com dados espectrais de $\mathrm{RMN}{ }^{1} \mathrm{He}{ }^{13} \mathrm{C}$ de 6 . Desta forma, a estrutura de 7 foi elucidada como sendo (7R*)-5-epi-oposit-4(15)-eno-1 $\beta, 7$ diol (7), que corresponde a uma substância que está sendo descrita pela primeira vez.

\section{CONCLUSÃO}

O estudo químico de sementes de um espécime de Guarea guidonia coletadas no distrito de Palmeiras, município de Dois Irmãos do Buriti - MS, levou ao isolamento de sete sesquiterpenos (1-7). As substâncias $6 \alpha$-etoxieudesm-4(15)-en-1 $\beta$-ol (1) e (7R*)-5-epioposit-4(15)-eno-1 $\beta, 7$-diol (7) são inéditas, enquanto eudesm-4(15)eno-1 $\beta, 6 \alpha$-diol (2) foi obtido em estudos anteriores realizados com cascas G. guidonia. Este é o primeiro registro de isolamento dos sesquiterpenos 5-epi-eudesm-4(15)-eno-1 $\beta, 6 \beta$-diol (3), eudesm$4(15)$-eno-1 $\beta, 5 \alpha$-diol (4), eudesm-4(15),7-dien-1 $\beta$-ol (5) e (7R*)oposit-4(15)-eno-1ß,7-diol (6) em Meliaceae.

\section{MATERIAL SUPLEMENTAR}

Os espectros de RMN de ${ }^{1} \mathrm{H},{ }^{13} \mathrm{C}$, HSQC, HMBC, COSY, NOESY e J-resolved, de substâncias isoladas de sementes de Guarea guidonia, estão disponíveis gratuitamente em http://quimicanova.sbq.org.br, na forma de arquivo PDF.

\section{AGRADECIMENTOS}

À FUNDECT-MS, CNPq, CAPES, CPq-PROPP/UFMS pelo apoio financeiro, à MSc. U. M. Resende (DBI/UFMS) pela identificação do material botânico e à Dra. E. D. Rodrigues pela obtenção e auxilio na análise dos experimentos de RMN J-resolved ${ }^{1} \mathrm{H}-{ }^{1} \mathrm{H}$.

\section{REFERÊNCIAS}

1. Corrêa, M. P.; Dicionário das Plantas Úteis do Brasil e das Exóticas Cultivadas, Imprensa Nacional: Rio de Janeiro, 1984, vol. 2.

2. Garcez, F. R.; Núñez, C. V.; Garcez, W. S.; Almeida, R. M.; Roque, N. F.; Planta Med. 1998, 64, 79

3. Lukacova, V.; Polonsky, J.; Moretti, C.; Pettit, G. R.; Schmidt, J. M.; J. Nat. Prod. 1982, 45, 288

4. Lago, J. H. G.; Brochini, C. B.; Roque, N. F.; Phytochemistry 2002, 60, 333.

5. Garcez, W. S.; Garcez, F. R.; Soares, L. R.; J. Braz. Chem. Soc. 2008, 19, 1073.

6. Furlan, M.; Lopes, M. N.; Fernandes, J. B.; Pirani, J. R.; Phytochemistry 1996, 41, 1159.

7. Brochini, C. B.; Roque, N. F.; J. Braz. Chem. Soc. 2000, 11, 361.

8. Lago, J. H. G.; Brochini, C. B.; Roque, N. F.; J. Essent. Oil Res. 2005, $17,271$.

9. Nagashima, F.; Kishi, K.; Hamada, Y.; Takaoka, S.; Asakaw Y.; Phytochemistry 2005, 66, 1662.

10. Zhang, H.; Tan, G. T.; Santarsiero, B. D.; Mesecar, A. D.; Hung, N. V.; Cuong, N. M.; Soejarto, D. D.; Pezzuto, J. M.; Fong, H. H. S.; J. Nat. Prod. 2003, 66, 609 .

11. Iijima, T.; Yaoita, Y.; Kikuchi, M.; Chem. Pharm. Bull. 2003, 51, 545.

12. Ma, K.; Zhang, C.; Liu, M.; Chu, Y.; Zhou, L; Hu, C.; Ye, D.; Tetrahedron Lett. 2010, 51, 1870.

13. Sun, Z.; Chen, B.; Zhang, S.; Hu, C.; J. Nat. Prod. 2004, 67, 1975.

14. Botrel, P. P.; Pinto, J. E. B. P.; de Araujo, A. C. C.; Bertolucci, S. K. V.; Figueiredo, F. C.; Ferri, P. H.; da Costa, D. P.; Quim. Nova 2010, 33, 33.

15. Xie, W. D.; Niu, Y. F.; Lai, P. X.; Row, K. H.; Chem. Pharm. Bull. 2010 , $58,991$.

16. Wei, H. H.; Xu, H. H.; Xie, H. H.; Xu, L. X.; Wei, X. Y.; Helv. Chim. Acta 2009, 92, 370. 